\title{
Study And Characterization Of Nanostructure Plasma Polymerized Aniline Thin Films
}

\author{
Rajendra Kumar* ${ }^{1}$, Ashish Kumar shukla ${ }^{1}$ \\ ${ }^{1}$ Department of Physics, Faculty of Engineering \& Technology, Rama University, \\ Kanpur-209217, U.P. INDIA \\ *Email: rajendrab25@gmail.com,ashukla.26jan@gmail.com
}

\begin{abstract}
In this work, we have to prepared plasma polymerized aniline thin films. Plasma polymerization is a process of synthesis, in which crystal are changed in nanostructures. We have to monitor the growth rate of thin films with help of pulses and established an ideal condition. The characterization of PANI thin film was performed with the ultraviolet-visible spectroscopy (UV-Vis), Fourier transform infrared spectroscopy (FTIR) and scanning electron microscope (SEM) studies. The surface of the thin film possessed three-dimensional networks of the PANI belts as cauliflower like morphology. In PANI prepared through chemical route, however no aniline seems to exist. The PANI films prepared by plasma polymerization are less crystalline compared by chemical route. PANI films prepared by plasma technique are however totally free from reagent chemicals. The sufficient sensitivity could be obtained for plasma prepared PANI sensors; there may be no need to go through cumbersome chemical route.
\end{abstract}

Keywords- Plasma polymer; thin Film; Nanostructure polyaniline.

\section{INTRODUCTION}

A wide range of the conducting polymers including polyaniline [1-8], polypyrrole[4] and polyacetylene [9] has been investigated through plasma polymerization. Among the reported advantages of plasmapolymerized materials are denseness, uniformity, controllable film thickness, adhesion and conformability on various substrates [10]. A wide range of volatile organic compounds can be used as the feed to a plasma reactor.But because no oxidants or solvents are involved, contamination of the film by extraneous species is eliminated in principle[11], Further the polymerization process requires only a single step. The composition of a plasma-polymerized film can be tailored with the appropriate processing conditions, and the resulting material properties are often unique and unobtainable by wet synthetic methods ${ }^{5}$. Although the excited plasma species are mainly high-energy electrons that are relatively indiscriminate in rupturing chemical bonds, the substrate can be kept at the ambient temperature [12]

Over all, plasma polymerization is an inexpensive processing system. PANI is the most highly conductive of all known polymers and has notably complex structureproperty behavior involving four different oxidation states; the conducting form is stable in both air and water ${ }^{13}$. In situ doping with iodine vapor [6] can modify the conductivity of plasma-polymerized PANI; subsequent ion implantation of $\mathrm{I}^{+}$in PANI films has also been reported [14]. The protonic doping from acidic solutions is also well known. The morphology of a PANI film depends on the method of preparation [15], the substrate [7], and the deposition time [7]. On glass, plasma-polymerized PANI first forms irregular particles, then clusters, and finally a continuous film of irregular pentagons; in contrast, on a Pt substrate, the initial spongy, spherical particles grow into fibrils [7]. The range of applications for PANI films includes sensors [16], corrosion protection[13] and electro chromic displays [11].

PANI can be synthesized by chemical, electrochemical, or plasma methods, or vacuum deposition methods and in each case, the composition, morphology, and physical properties of the resulting polymer are strongly dependent on the detailed reaction conditions.

Plasma polymerized aniline are IR spectroscopy [16], ultraviolet-visible spectroscopy [14], electron spin resonance [2], X-ray diffraction [4], thermal analysis, scanning electron microscopy (SEM) [15], elemental composition by electron spectroscopy [15],X-ray photoelectron spectroscopy[14], energy-dispersive spectroscopy/scanning electron microscopy [16], and the measurement of various electrical [14], and optical properties and also the contact angle [15].

PANI samples prepared by the wet-chemical or electrochemical oxidation of aniline have been examined by a similar range of dry ex situ methods. These include IR [19], UV-Vis spectroscopy [19], X-ray diffraction [19], CNMR[18], scanning tunneling microscopy [21], and atomic force microscopy[19].

Quantitative and qualitative wet analytical methods have also been applied to these polymers. Methods include gel permeation chromatography[19], solubility determination, and various electrochemical [21] and spectroelectrochemical [23], techniques. Wet methods have seldom been used in characterizing plasma-polymerized materials. Although it is true that immersing a previously dry polymer film is likely to cause irreversible changes in its properties, invaluable information can be gained that permits a much more detailed comparison of materials made by different process routes.

\section{PREPARATION OF PANI THIN FILMS}

With the help of inductively coupled pulsed plasma reactor process, we have to prepared the polyaniline thin films. Independently of the pulsing of the RF plasma process, the monomer injector also operates in a pulsed manner; the RF frequency is much greater than that of the injector, so each burst of injected aniline is exposed to a plasma reactor environment that is effectively steady. The pulsed-plasma reactor system having monomer injection system with custom-built automotive fuel injector, a syringe containing liquid monomer under pressure, and an oscilloscope to provide a timing pulse to control the injection of vaporized monomer (Fig.1). When a pulse of the monomer entered 


\section{Available online at www.ijrat.org}

the evacuated reactor, most of the liquid immediately vaporized by flash boiling, any remaining liquid disintegrated into droplets that were collected by mesh separators placed about $2 \mathrm{~cm}$ from the injector nozzle. The aniline was injected in 10-ms pulses until the pressure in the reactor reached the desired value at $40 \mathrm{~Pa}$.

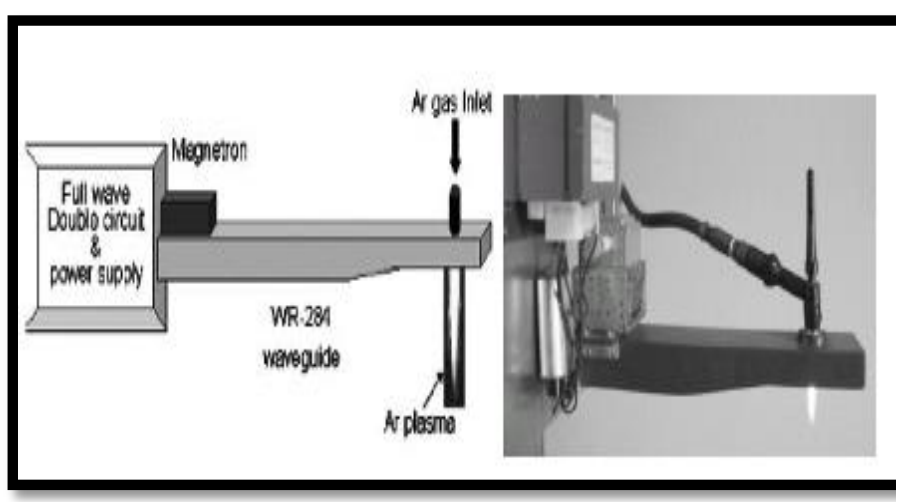

Figure.1 A typical picture of plasma polymerization unit.

\section{EQUIPMENTAL:}

The monitoring of the reactor pressure was done with a thermocouple gauge and a capacitance monometer. The manometer was used to calibrate the thermocouple gauge in the absence of plasma and was removed during film deposition. Calibration runs were performed in triplicate from 0 to $1000 \mathrm{~m}$ Torr for air, aniline vapour, and a mixture of aniline vapour and hydrogen. When enough monomer had been injected into the reactor to reach the desired deposition pressure, the plasma was repetitively activated through the discharge of a $1.8-\mu \mathrm{F}$ capacitor initially held at $23 \mathrm{kV}$, the RF coil excitation being with a damped sinusoid of $290 \mathrm{kHz}$ and a decay time constant of $10 \mu \mathrm{s}$. After 10 such plasma shots, the reactor was evacuated completely and refilled with fresh monomer vapour. One hundred plasma pulses were used to grow most PANI samples. The substrate holder could be positioned at various distances from the RF coil. The substrates included glass plates, indium tin oxide (ITO) glass, and glass microscope slides; in some runs, the choice of the substrate was dictated by the characterization method that was to be used. The reagent-grade aniline monomer was purified by triple distillation over zinc granules and then stored in a dark bottle under nitrogen [13].

\section{RESULTS AND DISCUSSION:}

The average deposition rate obtained for different plasma processes and number of pulses involved to prepare a film is discussed as in the following.

\subsection{Film Deposition Rate}

Variations in the film thickness can provide a direct measure of the net growth rate under different conditions. The profilometer data can directly be correlated with the pressure in the reactor and the number of plasma pulses, but the growth dynamics is complex. The average growth rate increases with higher pressure for a fixed number of plasma pulses, but at pressures of 27 or $40 \mathrm{~Pa}$, the average rate decreases as the number of pulses is raised from 50 to 100 . The drop is more pronounced at higher pressure. At $13 \mathrm{~Pa}$, however, the deposition rate increases if more pulses are used. Different mechanisms of polymerization or film growth may be rate-controlling under different conditions ${ }^{29}$ and there may be a critical film thickness at which the growth rate slows down.

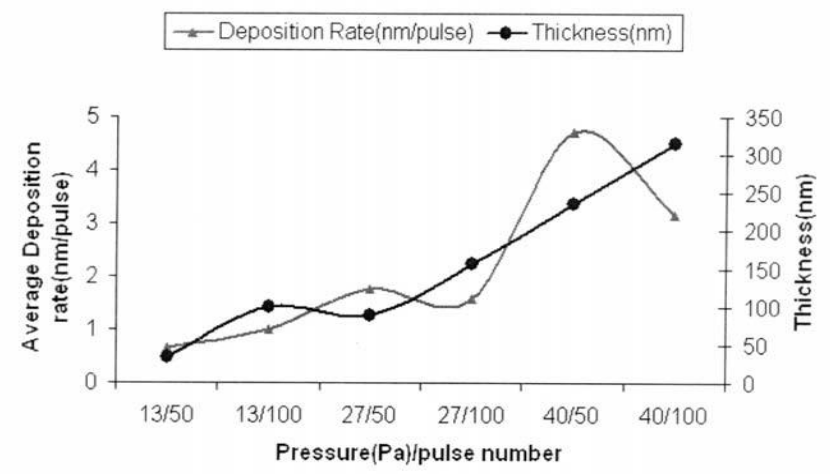

Figure 2. Film thickness and average deposition rate of nanofibrous PAN thin film.

\section{CHARACTERIZATION OF PANI FILM:}

The prepared PANI thin films were characterized by using UV-Vis, FTIR, XRD and SEM. An Ocean Optics HR 4000 High Resolution Spectrophotometer recorded the UV-Vis spectra of the films. For FTIR measurements the films were grown on glass microscope slides and then were scraped off and pressed into $\mathrm{KBr}$ pellets. The spectra were recorded between 4000 and $400 \mathrm{~cm}^{-1}$ with a Perkin Elmer BX FTIR microscope (Shelton, CT); an averaging of 64 scans was done to minimize noise. The XRD spectra of the material were obtained by using powder XRD system Model PAN analytical X'Pert Pro having $\mathrm{CuK}_{\alpha 1} \mathrm{X}$-ray source of wavelength $1.5406 \AA$. The sample morphology and surface uniformity were assessed microscopically with SEM (S-570, Hitachi, and San Jose, CA) in the secondary electron mode. Finally the results obtained for the present PANI samples have been compared with those available for PANI samples prepared by chemical methods.

\section{1. $U V$-Vis Spectra}

The UV-Vis spectrum of the PANI thin film grown on glass substrate (Fig.3) shows an absorption peak in the UV range at $328 \mathrm{~nm}$ and another in visible at $560 \mathrm{~nm}$. These are characteristic peaks of undoped PANI. The peak at $328 \mathrm{~nm}$ corresponds to $\pi-\pi$ excitations of amine nitrogen of the benzenoid segments and that at $560 \mathrm{~nm}$ to imine nitrogen of the quinoid segments of PANI respectively ${ }^{25}$. The observed UV-Vis spectra thus support the formation of PANI through plasma polymerization.

However, in the Fig 3.3 spectrum of PANI samples obtained through wet chemical method [26] the above two peaks were at $328 \mathrm{~nm}$ and $635 \mathrm{~nm}$ respectively. There was further an additional third peak at $268 \mathrm{~nm}$, which was associated to change transfer process. 
International Journal of Research in Advent Technology, Vol.7, No.4, April 2019

E-ISSN: 2321-9637

Available online at www.ijrat.org

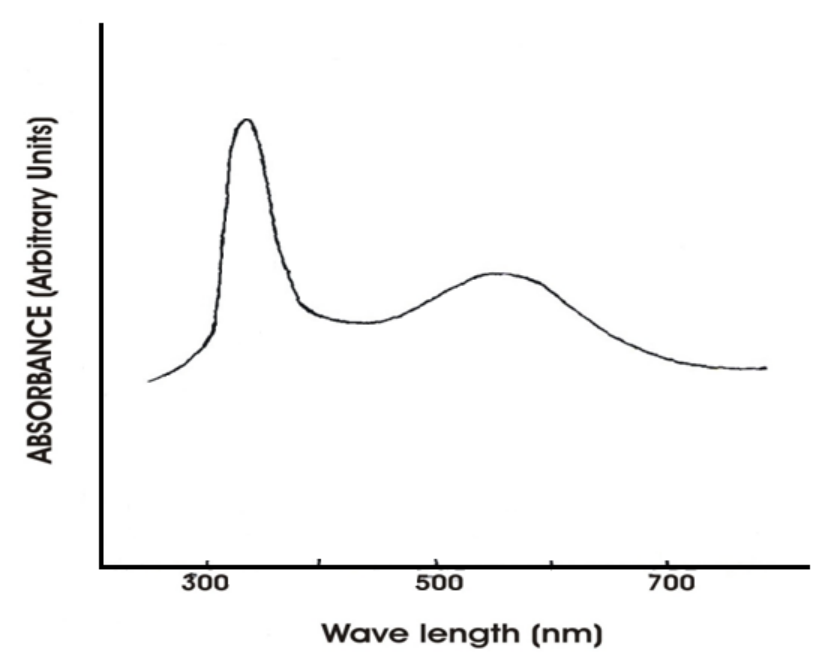

Figure 3. Uv-Vis spectrum of the PANI thin film..

\subsection{FTIR Spectra}

FTIR spectra (Fig) indicates not only that the chemical bonding in plasma-polymerized aniline differs from that of the monomer but also that the molecular structure of the deposited film is a strong function of distance $(\mathrm{z})$ from the $\mathrm{RF}$ coil in the plasma reactor. The strong $\mathrm{z}$ dependence of FTIR spectra and thus of chemical composition and structure of plasma-polymerized materials has long been known [27-28]. The films whose FTIR spectra are shown in Figure were grown on glass slides placed 16, 24, 32, and 40 $\mathrm{cm}$ from the center of the coil, under otherwise identical conditions (40 $\mathrm{Pa}$ and 100 plasma pulses). For an easy comparison, the spectrum of the aniline monomer is also shown in Figure. But it should be noted that the intensities of all five spectra are in arbitrary units and therefore are not directly comparable. The spectra for $\mathrm{Z}=40 \mathrm{~cm}$ is well marked and explained in the text.

We can determine the effect of the axial position ( $\mathrm{z}$ ) on the chemical functionality of the films by first identifying bands that are common to all four of the PANI spectra and then focusing on features that are significantly different. The common bands include those near $3338 \mathrm{~cm}^{-1}$, indicative of a secondary amine; those at $698 \mathrm{~cm}^{-1}$ and 756 $\mathrm{cm}^{-1}$, indicative of deformation in a monosubstituted benzene ring; and those at 439 and $541 \mathrm{~cm}^{-1}$, indicative of deformation of substituted benzene. However, the spectrum for the 40-cm film shows a clearer evidence for aromaticity than any of the others. The I. R. bands near 1027, 1069, $1156,1182,1448,1492,1539$, and $1599 \mathrm{~cm}^{-1}$ are present in the spectra for different Z- films but are considerably sharper for the $\mathrm{Z}=40 \mathrm{~cm}$ film; and further all are consistent with aromaticity. A comparision of the FTIR spectrum of Fig. 3 for plasma-polymerized aniline with the FTIR spectrum of PANI prepared through chemical route [Fig. 4] shows that there is a unique band at $3024 \mathrm{~cm}^{-1}$ in Fig. 3, which is totally absent in Fig. 3. This shows that during plasma polymerization some aniline monomer or its oligimers remain in the PANI film prepared. This band is more significantly seen in film prepared for low $\mathrm{Z}$ values, and seems to be due to aniline or its oligimers.

The spectra for films grown closer to the RF coil (smaller Z) have a prominent band in the region from 1673 to $1684 \mathrm{~cm}^{-1}$, which shows frequencies that are associated with $\mathrm{C}=\mathrm{O}$ stretching of a ketone created due to oxygen left in the reactor after pump down, Such a band may also be assume to $\mathrm{C}=\mathrm{C}$ band of quinoid ring. The $\mathrm{C}=\mathrm{C}$ bond of benzenoid rings has clearly been observed at $1492 \mathrm{~cm}^{-1}$. The relative intensity of this band decreases as the axial distance increases. These observations are consistent with those obtained by other workers regarding the plasma polymerization of aniline ${ }^{27}$ and fluorinated monomers ${ }^{29}$. It is believed that free radicals are trapped in the as-grown film in concentrations that increase with the field strength; it is however not clear whether these highly energetic species react in situ with residual oxygen left in the reactor after pump down or whether subsequent exposure to atmospheric concentrations of oxygen is required. It may be relevant that films handled initial way to low $\mathrm{Z}$ but grown at $Z=40 \mathrm{~cm}$ do not show any carbonyl band in FTIR. The model of electron temperature profiles developed earlier by Shepsis predicts that the plasma electrons should be most energetic in the vicinity of the RF coil and that their temperature should decrease with the axial distance away from it.

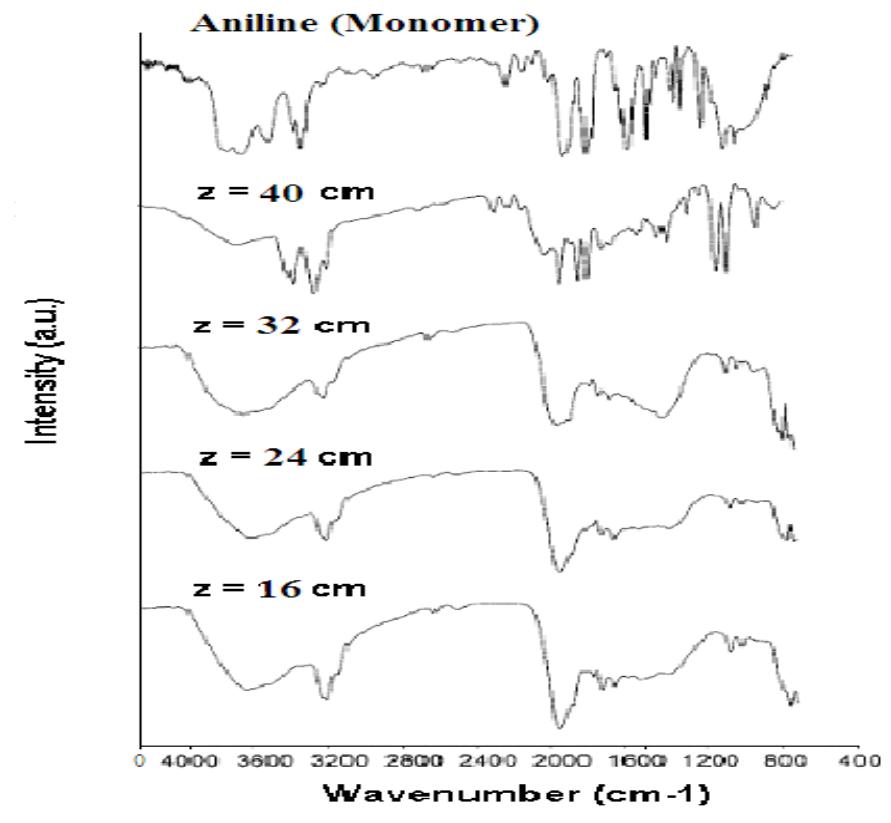

Figure 4. FTIR spectra of aniline monomer and plasma polymerized aniline films deposited on ITO substrates at various distances from the center of the RF coil.

\subsection{XRD Spectra}

The XRD of the PANI thin films prepared by plasma technique clearly shows their amorphous nature and gives unresolved crystalline peaks in the $15^{\circ}-30^{\circ}$ region of $2 \theta$ (Fig. 3.6). The XRD spectra of PANI prepared by chemical route are further shown in Fig. 5. These spectra show resolved broad band in $7^{\circ}-30^{\circ}$ region of $2 \theta$. A comparison of XRD spectra of Fig. shows that PANI crystallites are of smaller size $\left(<10^{-6} \mathrm{~cm}\right)$ in plasma polymerized films. The XRD peaks for PANI prepared by chemical route do exhibit some resolved structure for $2 \theta$ from $7^{\circ}$ to $30^{\circ}$, indicating that in such PANI samples the crystallites are of somewhat bigger size, due to enough crystallization in the process of preparation. 


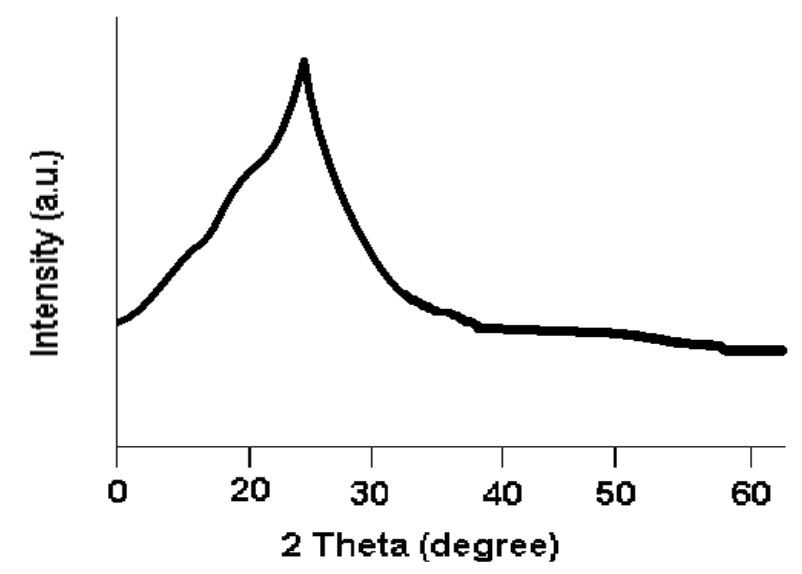

Figure 5. XRD spectra of polyaniline thin film prepared by plasma polymerization technique at $40-\mathrm{Pa}$ reactor pressure.

\subsection{SEM}

SEM images show that the morphology plasma polymerization of the PANI thin film and as per Fig.6 exhibit smooth texture in films. So a films prepared through chemical route are concerned, the SEM images were not much different from Fig

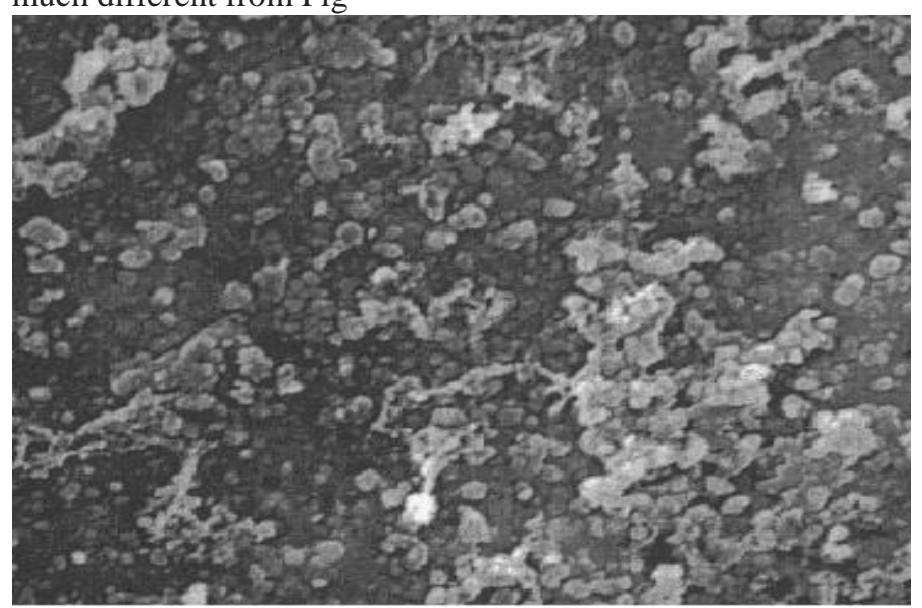

Figure 6. SEM micrographs of PANI deposited on glass substrate 40-Pa reactor pressure and 50 plasma pulses.

\section{CONCLUSIONS:}

UV-Vis, FTIR, and XRD spectra of plasma-polymerized films clearly indicate the existence of polyaniline. Some aniline monomer, oligimer content seems to exist in plasma prepared PANI. In PANI prepared through chemical route, however no aniline seems to exist. The PANI films prepared by plasma polymerization are less crystalline compared by chemical route. PANI films prepared by plasma technique are however totally free from reagent chemicals.

\section{ACKNOWLEDGEMENTS:}

Authors are thankful to the Director, National Physical Laboratory, New Delhi, India for providing infrastructure facilities to carry out this work and also thankful to Vice Chancellor, Rama University Utter Pradesh, Kanpur for providing Lab Facilities.

\section{REFERENCES:}

[1] L. V. Shepsis, P. D. Pedrow, R. Maralinga, M. A. Osman, "Modeling and experimental comparison of pulsed plasma deposition of aniline"Thin Solid Films. Volume 11, no. 385 pages 11-21, 2001.

[2] X. Gong, L. Dai, A. W. H. Mau, H. J. Griesser, "Plasma-chemical synthesis and properties of polymer semiconductors and prospectsfor their use" J Polym Sci Part A: Polym Chem. Volume 36, pages 633- 643, 1998.

[3] M. G Olayo, J. Morales, G. J. Cruz, R. Olayo, ez, E. Ordon, S. R. Barocio, "Polymerization of halogenated anilines by plasma" J Polym . Sci Part B: Polym Phys. Volume 39, pages 175, 2001.

[4] J. Morales, M. G. Olayo, G. J. Cruz, M. M. CastilloOrtega, R. Olayo, "Electronic conductivity of pyrrole and aniline thin films polymerized by plasma" J. Polym. Sci Part B: Polym Phys. Volume 38, pages 3247-3255, 2000.

[5] C. J. Mathai, S. Saravanan, M. R Anantharaman,. S. Venkitachalam, S. Jayalekshmi, "Characterization of low dielectric constant polyaniline thin film synthesized by ac plasma polymerization technique" J. Phys D: Appl. Phys. Volume 35, pages 240-245, 2002.

[6] G. J. Cruz, J. Morales, M. M. Castillo-Ortega, R. Olayo "Synthesis of polyaniline films by plasma polymerization" Synth Met. Volume 88, pages 213218, 1997.

[7] N. V. Bhat, N. V. Joshi, "Structure and Properties of Plasma-Polymerized Thin Films of Polyaniline" Plasma Chem Plasma Proc. Volume 14, pages 151-161, 1994.

[8] M. Augestine, M. Sebastian, K. A. Malini, M. J. S. Joseph, T. Joseph, D. Sakthikumar, S. Jayalakshmi, T. M. S. Rasheed, M. R Anantharaman,."Preparation and spectroscopic characterization of plasma polymerised poly aniline thin films" Indian J Pure Appl Phys. Volume 34, pages 966-971, 1996.

[9] K. O. Goyal, R. Mahalingam, P. D Pedrow, M. A. Osman, "Mass transport characteristics in a pulsed plasma enhanced chemical vapor deposition reactor for thin polymer film deposition" IEEE Trans Plasma Sci. volume 29, pages 42-50, 2001

[10] Han, L. M. Timmons, R. B. "Pulsed-plasma polymerization of1-vinyl-2-pyrrolidone: Synthesis of a linear polymer" J Polym Sci Part A: Polym Chem. Volume 36, pages 3121-3129, 1998.

[11] G. J. Cruz, J. Morales, R Olayo, "Films obtained by plasma polymerizationof pyrrole" Thin Solid Films. Volume 342, pages 119-126, 1999.

[12] R. K. Sadhir, Jr. Schoch, "Preparation and properties of plasma- polymerized thiophene (PPT) conducting films" Thin Solid Films. Volume 223, pages 154-160, 1993.

[13] D. E. Stilwell, S.-M. Park, "Electrochemical Studies on Growth Properties of Polyaniline" J Electrochem Soc. A, Volume 135, pages 2254-2262, 1988.

[14] Z. S Tong,. M. Z. Wu, T. S Pu,. F. Zhou, H. Z. Liu, "Plasma induced aniline polymerization" Synth Met. Volume 68, pages 125-131, 1995. 


\section{Available online at www.ijrat.org}

[15] R. Hernandez, A. F. Diaz, R. Waltman, J. Bargon,

"Electrochemically Induced Volume Changes in Poly (3, 4ethylenedioxythiophene)" J. Phys. Chem. Volume 88, pages 3333-3337, 1984.

[16] S. Takeda,. "A new type of CO2 sensor built up with plasma polymerized polyaniline thin film "Thin Solid Films.volume 313, pages 343-346, $1999 .$.

[17] D. M. Mohilner, R. N Adams. W. J. Argersinger, Jr. "Properties of cyanidefunctionalized polyaniline prepared by oxidative electrochemical polymerization of $o$ aminobenzonitrile" J Chem. Soc. Volume 84, pages 3618-3662, 1962.

[18] A. P. Monkman, P. Adams, "Structural characterisation of polyaniline frees standing films" Synth Met. Volume 41, pages 891896, 1991.

[19] M. Angelopoulos, R. Dipietro, W. G. Zhang, A. G. MacDiarmid, A. Epstein"Effect of selected processing parameters on solution properties and morphology of polyaniline and impact on conductivity" J. Synth. Met. Volume 84, pages 34-39, 1997.

[20] D. E. Stilwell, S.-M Park, "Electrochemistry of Conductive Polymers: IVElectrochemical Studies on Polyaniline Degradation - Product Identification and Coulometric Studies" J Electrochem Soc. Volume 135, pages 24972502, 1988.

[21] H. Yang, A. J. Bard, "The application of fast scan cyclic voltammetry. Mechanistic study of the initial stage of electropolymerization of aniline in aqueous solutions" J Electroanal Chem. Volume 339, pages 423-449, 1992.

[22] G. A. Mabbott, "An introduction to cyclic voltammetry" J Chem Educ. Volume 60, pages 697-702, 1983.

[23] D. E. Stilwell, S.-M. Park, "Electrochemistry of Conductive Polymers: III Some Physical and Electrochemical Properties Observed from Electrochemically Grown Polyaniline" J Electrochem Soc. volume 135, pages 2491-2496, 1988.

[24] L.V. M.S. Shepsis, Thesis, Washington State University, 1999

[25] M. Scully \& M. C. Petty, "Optical properties of polyaniline thin films" Synthetic Metals, volume 55, pages 183-187, 1993.

[26] P. Swami (unpublished work).

[27] D. W Rice, D. F. O'Kane, “Glow Discharge Polymerization of Tetrafluoroethylene, 1,1 Difluoroethylene, and Chlorotrifluoroethylene" J Electrochem Soc.volume 123,pages 1308-1312, 1976.
[28] D. F. O'Kane, D. W. Rice, "Preparation and Characterization of Glow Discharge Fluoropolymer-Type Polymers " J Macromol Sci Chem. A Volume 10, pages 567-577, 1976.

[29] R., Kumar, S., Singh, \& A.K., Mishra, "Electrical, Optical and Structural Characterization of Polyaniline Thin Film Prepared by Pulsed-plasma Polymerization" J. Polym. Mater. Voume. 27, pages 369-377, 2010, 